\title{
Clay fine fissuring monitoring using miniature geo-electrical resistivity arrays
}

\begin{abstract}
This article describes a miniaturised electrical imaging (resistivity tomography) technique to map the cracking pattern of a clay model. The clay used was taken from a scaled flood embankment built to study the fine fissuring due to desiccation and breaching process in flooding conditions. The potential of using a miniature array of electrodes to follow the evolution of the vertical cracks and number them during the drying process was explored. The imaging technique generated two-dimensional contoured plots of the resistivity distribution within the model before and at different stages of the desiccation process. The change in resistivity associated with the widening of the cracks were monitored as a function of time. Experiments were also carried out using a selected conductive gel to slow down the transport process into the cracks to improve the scanning capabilities of the equipment. The main vertical clay fissuring network was obtained after inversion of the experimental resistivity measurements and validated by direct observations.
\end{abstract}

Keywords Resistivity - Clay cracking · Desiccation . Embankments

\section{Introduction}

Long term deterioration is recognised to be an important factor affecting the integrity and reliability of flood embankments. Changes in material properties due to desiccation or softening of clays as well as changes in the structural form of embankments caused by erosion and/or burrowing can affect the full structure. The process of desiccation fissuring is known to contribute to embankment failure during overflow conditions that can lead to excessive ingress of water into the crest and on the outward slope ending into breaching and slope failure. However, very little information is known about the rate of desiccation and the cracking network within newly constructed flood embankments. Likewise no robust methods have been developed to monitor the presence of fissures except than excavating trial pits for inspection.

The fine fissuring of clay fill was first recognised as a major cause of flood embankment failure in the UK following the devastating North Sea floods of 1953. Cooling and Marsland (1954) carried out extensive field studies of the areas affecting by flooding in Essex and Kent and concluded that embankment failure was a result of one or a combination of the following causes: (1) erosion of the outward face by wave action, (2) erosion of the inward face due to overflow, (3) slipping of the inward face caused by seepage through the embankment, (4) build up water pressures in underlying permeable strata resulting in uplift.

Alternations in weather patterns due to climate change (i.e. increased rates of evaporation due to drier summers coupled with increase in heavy rainfall and extreme flood events) have induced a greater likelihood of long term desiccation of flood embankments with the possibility of more frequent extreme flood events like in 2003. Marsland (1968) carried on the study in investigating the fissuring of clay in flood embankments.

Therefore, the aim of the present study was to investigate the evolution of the desiccation process using a miniature geo-electrical method in order to map the 
vertical fissuring network and number the cracks in the clay used to build a scaled embankment in the hydraulic laboratory of the Civil Engineering Department. Further experiments will be carried out on the field to explore cracks on real embankments.

Clay soils tend to crack when they dry. However, the precise mechanism of cracking in not perfectly understood. Factors that influence cracking are known qualitatively, but it is not clear how to predict the depth of cracking and the spacing of cracks. Konrad and Ayad (1997) proposed an idealized framework for the analysis of cohesive soils undergoing desiccation. This has led to a simplified model referred to as CRACK, which enables the prediction of the average spacing between primary cracks for a homogeneous soil deposit subjected to a given surface evaporation flux. Konrad and Ayad (1997) presented the results of a field desiccation experiment in a top soil, a weathered crust, and an intact clay deposit at the experimental site of Saint-Alban, Quebec, Canada. The excavation in the intact clay was instrumented to monitor the evolution of different parameters such as suction, moisture content, settlement, temperature, and relative humidity with time while the evolution of surface cracking was recorded using photographs. Desiccation of the intact Saint-Alban clay under restrained conditions, at an average evaporation rate of $0.018 \mathrm{~cm} / \mathrm{h}$ and an initial gravimetric water content of $103 \%(\mathrm{~kg} / \mathrm{kg})$, produced visible primary cracks after $17 \mathrm{~h}$ with an average spacing of 20-24 cm. The average size of the major polygonal blocks ranged between 20 and $24 \mathrm{~cm}$. After $27 \mathrm{~h}$ of evaporation, the average opening between cracks was about $1.5 \mathrm{~mm}$, and after $68 \mathrm{~h}$ it was $4 \mathrm{~mm}$, indicating an ongoing volume shrinkage.

The results from Rayhani et al. (2007) showed that the dimension of cracks increased with increasing plasticity index and clay content and so, the initial hydraulic conductivity increased with increasing plasticity index and cycles of drying and wetting.

Nahlawi and Kodikara (2006) carried out some experiments on the induced cracking tests on thin layers of clay soils using humidity and temperature controlled chamber for observation, crack initiation and evolution and for moisture content measurement during desiccation. The lengths of the moulds were considerably larger than their widths so that parallel cracking were generated in thin layers.

Lecocq and Vandewalle (2003) used a simple onedimensional experiment to investigate the dynamical aspects of crack opening that occurs in clay exposed to shrinkage induced by desiccation. The opening rate of cracks was a varying (diminishing) function of time. They observed a strong influence between the rate of apparition of a new crack and existing cracks. As soon as the second crack appears, the opening rate of the first crack falls by half. The mechanism of clay cracking has been well documented by other authors (Towner et al. 1987; Moore 1984; Chertkov and Ravina 1998; Augier et al. 2002) but the identification of the subsurface cracking network is still under-estimated especially for embankments.

Geophysical methods based on miniature resistivity arrays could be the solution as they have proved to be reliable to monitor contaminant transport in soils scaled models in centrifuge experiments (Depountis et al. 1999). This technique is non-invasive and hence is reducing considerably the disturbance of the soil, improving the accuracy of the measurements.

For the assessment of embankments this technique could be very useful for long term monitoring of clay sealings which are not accessible.

Although the technique presented here may not have the accuracy of a local sampling strategy and laboratory testing, subsurface tomography is valuable for interpretation in heterogeneous soils.

The subsurface soil properties are determined by measuring the distribution of resistivity. The basis of the technique is to pass a direct current through the soil between a pair of electrodes. This process is observed by monitoring the distortion of the equipotentials (assuming the soil to be a homogeneous half-space) using another pair of potential electrodes located at the ground surface (Barker 1997). This provides a simple, repeatable technique that can be applied where any contrast in electrical conductivity exists in space (or time). Lataste et al. (2003) used the resistivity technique with a device made of four electrodes spaced out 5 or $10 \mathrm{~cm}$, arranged in a square to study the cracks on a damage concrete slab. He used a numerical modelling approach rather than an inversion model showing a qualitative similar disturbance of apparent resistivity right to cracks, for depth or opening variations. On a wider scope at geology level, Nguyen et al. (2005) proposed a methodology to locate automatically limits or boundaries between synthetic faults and layer boundaries in two dimension electrical tomography using a crest line extraction process in gradient images. He found that the method showed poor results when vertical gradients are greater than horizontal ones but otherwise should be systematically used to improve tomography interpretation.

In most of the geo-electrical surveys the resistivity technique usually involves a computer controlled multielectrode arrays to give a tomography contour model of the subsurface in two and three dimension (Griffiths and Barker 1993). One of the first team to use miniature resistivity imaging to detect cracks of $\mathrm{cm}$ size was Samouëlian et al. (2003). They used porous special electrodes filled with $\mathrm{CuSO} 4$ similar to the one currently used for self potential measurements to improve the electrical contact in creating a wet contact with the surrounding dried soil. They created 
artificially a crack of $2 \mathrm{~mm}$ width with a saw at varying depths $(1,2,3$, and $4 \mathrm{~cm}$ deep) in order to obtain four cracking stages. The highest interpreted electrical resistivity was detected in the top $1.5-\mathrm{cm}$ depth of their soil sample, whereas the crack developed down to $4 \mathrm{~cm}$. The electrical images obtained from these electrodes enabled the detection of structures at the millimeter scale. More recently Tabbagh et al. (2007) developed a new inversion model for assessing and simulating the electrical response and the main physical parameters of cracks in soils. Their model allowed a faster inversion of the experimental results. Rather than recreating artificially cracks and improve the inversion model, the present research has focused on the natural apparition of cracks and their direct detection using resistivity arrays and then using viscous tracers.

\section{Experimental procedure}

\section{Material}

Most of UK flood embankments are constructed as homogenous and impermeable bodies. The type of material used for the construction varies with the location and especially with the geological deposit available close to the planned construction site. In UK clay is mainly used as the fundamental fill, and plays a major role in the embankments design.

The soil chosen for this experiment is called Boulder Clay, and has been used to construct a flood embankment in the village of Galston, on the south-west from Glasgow, UK, as flood prevention.

According to the British Standards, a series of tests have been carried out to investigate the used clay properties, like: Attenberg Limits, Standard Proctor Method and linear shrinkage.

The maximum dry density for Galston clay has been found as $1.95 \mathrm{Mg} / \mathrm{m}^{3}$ at an optimum moisture content of $12 \%$. Particle dry density was found as $2.58 \mathrm{Mg} / \mathrm{m}^{3}$. Liquid and plastic limit for tested clay, were found as 35.7 and $16.4 \%$ with the Plasticity Index to be $36.2 \%$. Linear shrinkage was defined from the average of 9 samples, to be $10.13 \%$.

The undrained shear strength has been found to be $70 \mathrm{kPa}$, and soil was classified as firm clay, according to the British Standards 8004:1986.

\section{Experimental set up}

One hundred and ninety kilograms of clay was oven dried to remove all the moisture and sieved using $20 \mathrm{~mm}$ mesh sieves. The sample was mixed with water to obtain $15 \%$ of soil moisture content. Then it was left for $24 \mathrm{~h}$ for a curing period. The chosen value of moisture content was within the range of moisture for $95 \%$ of maximum dry density, and was close to the natural moisture content measured in the field.

After the soil preparation, a $2.5 \mathrm{~kg}$ compaction load was applied to several layers $5 \mathrm{~cm}$ deep, over the $1.5 \mathrm{~m}$ length, $0.25 \mathrm{~m}$ wide and $0.4 \mathrm{~m}$ high Perspex tank, secured by 3 steel clamps. The transparency of the Perspex tank allowed checking the uniformity of the clay compaction.

\section{Miniature resistivity array}

To identify the soil layers location already known, a miniature resistivity array was adapted to be used with the ARES earth meter equipment purchased from the company Gf instruments. Forty eight non-corrosive $1.5 \mathrm{~mm}$ diameter and $6 \mathrm{~cm}$ long electrodes (see Fig. 1) were wired up and connected with the automatic resistivity system using double 24 ways connectors.

The electrodes were pushed $3 \mathrm{~cm}$ into the compacted clay keeping a $3 \mathrm{~cm}$ spacing between them. To initiate and perform the desiccation and drying process a $1.2 \mathrm{~kW}$ infrared heater was placed $0.9 \mathrm{~m}$ above the clay surface, as shown in Fig. 2.

The measurements selected option was a two-dimensional multi-electrodes resistivity profile. A WennerSchlumberger array profiling method as shown in Fig. 3, was chosen in this study because it is the most sensitive configuration to vertical resistivity changes (horizontal structures) in the soil strata and the groundwater table, and it is also more sensitive than other arrays to the horizontal resistivity changes (vertical structures). Furthermore, the extensive horizontal coverage and greater number of data points than other arrays justified its choice.

In the case presented here, only the first four levels and 168 plotting points were chosen for data analysis in relation to the measurements taken outside the physical boundaries

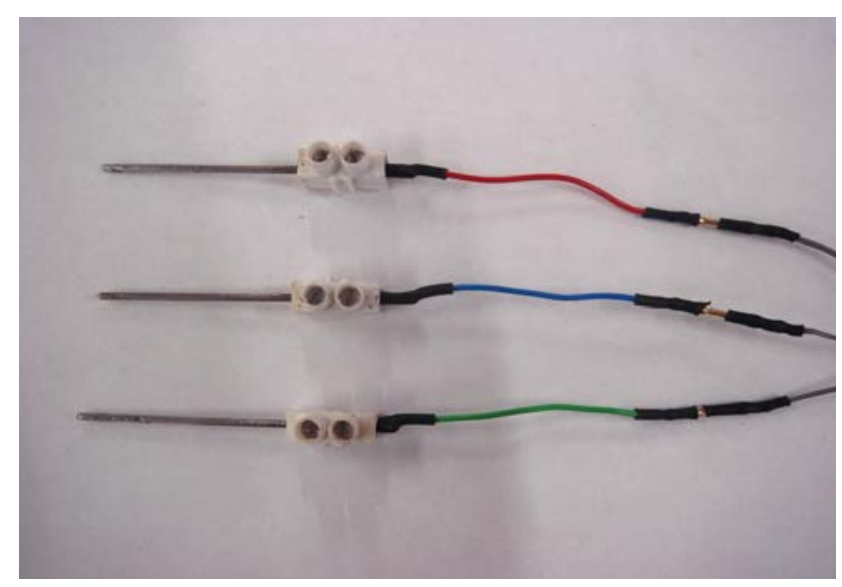

Fig. 1 Non-corosive electrodes mounted in block terminals 


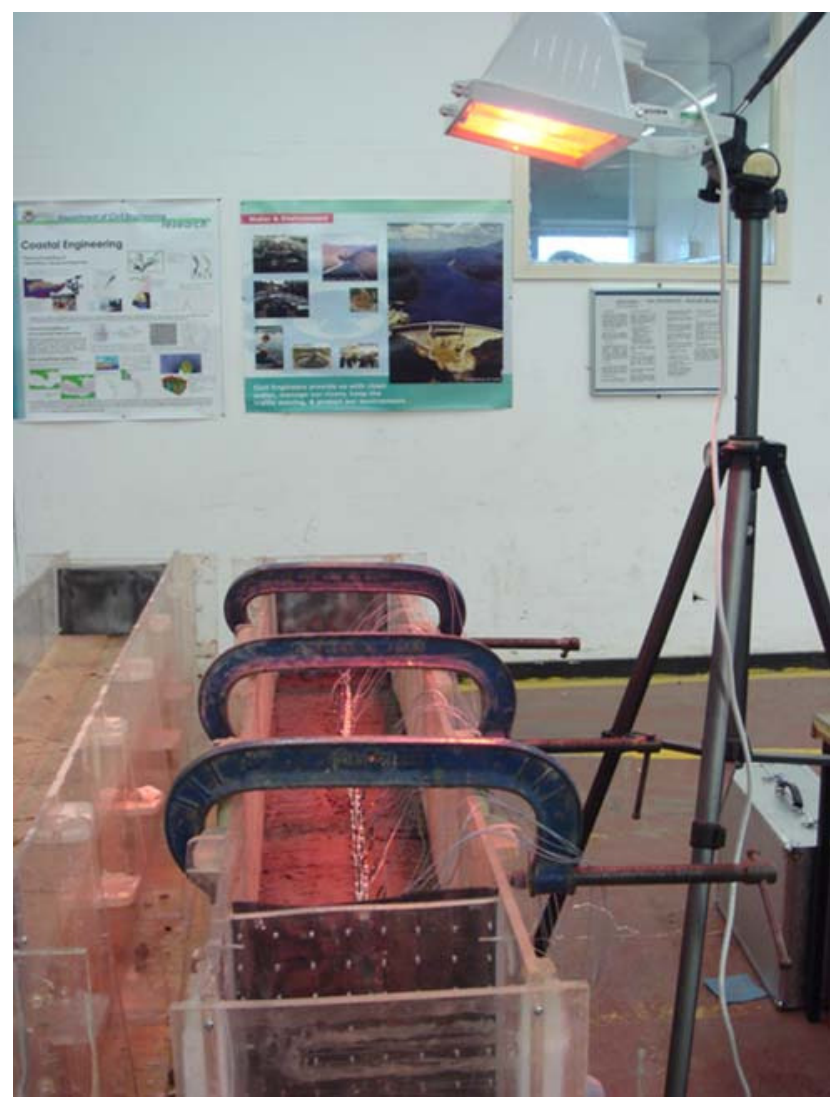

Fig. 2 Experimental setup with infra-red heater and electrodes installed

(depth) of the flume model, which were identified as high resistivity measurements due to the plastic interferences at the bottom of the Perspex tank.

An experiment was carried out with the flume tank only filled with water with a measured conductivity of $63.2 \mu \mathrm{s} / \mathrm{m}$ to investigate the geometry of outer boundaries and electrodes. Figure 4 shows the geo-electrical profile obtained. It

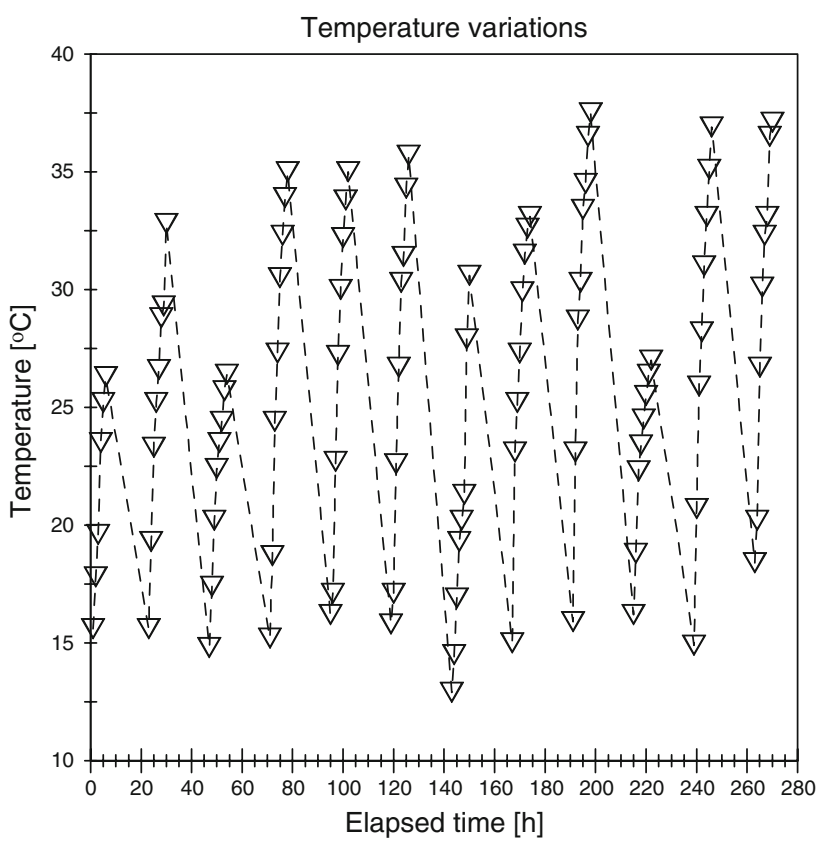

Fig. 5 Temperature variations for 12 days drying process

can clearly be seen the resistivity artifact due to the bottom Perspex tank boundaries in deep purple and the water in deep blue.

The experiments were carried out for 12 days. Every morning the infra-red heater was switched on and left for $6 \mathrm{~h}$ to initiate and perform desiccation cracking. During this drying stage the variations in soil temperature were recorded every hour. Then the geo-electrical scan was carried out, and the equipment was switched off overnight. The initial temperature of the soil in the morning was recorded at $20 \mathrm{~cm}$ depth every day before starting a new drying stage. Each last measurement was taken after drying has been finished (see Fig. 5).

Fig. 3 Four levels array of plotting points chosen for data analysis
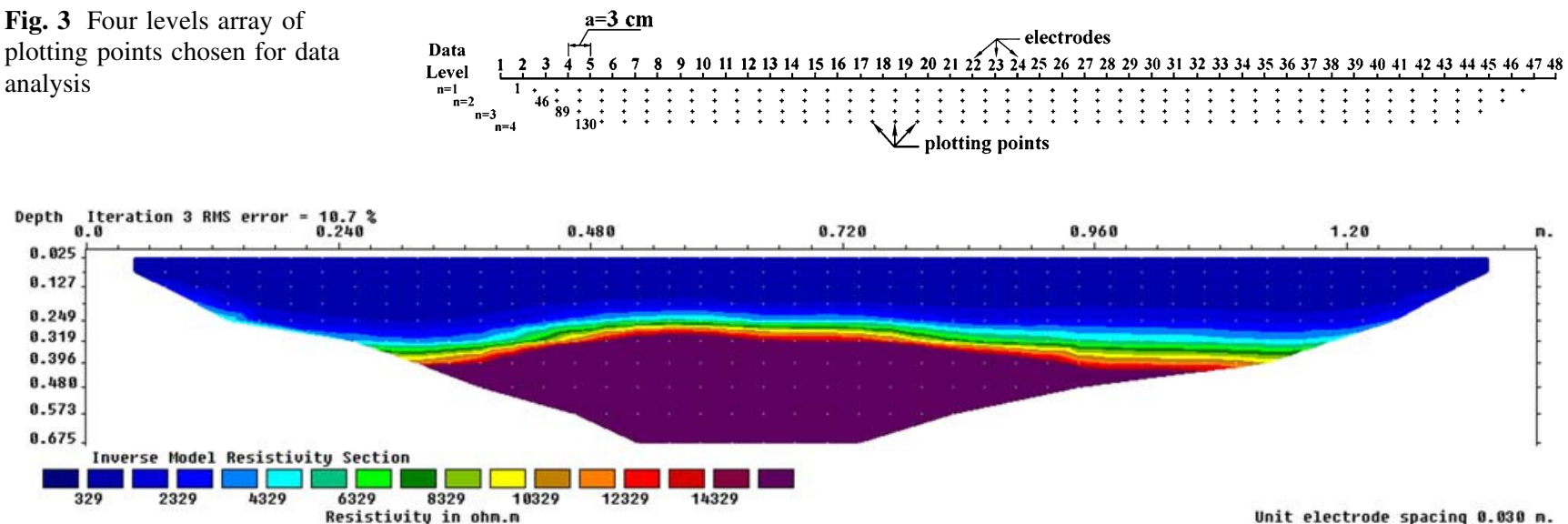

Unit electrode spacing $0.030 \mathrm{n}$.

Fig. 4 Water and tank boundaries resistivity profile, baseline 


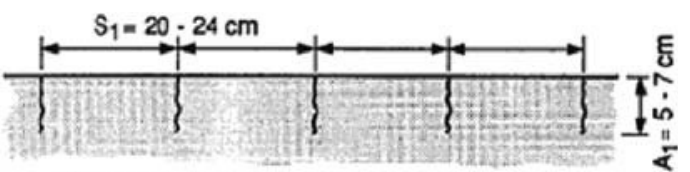

a) Initiation of primary cracks for $t<17 \mathrm{~h}$

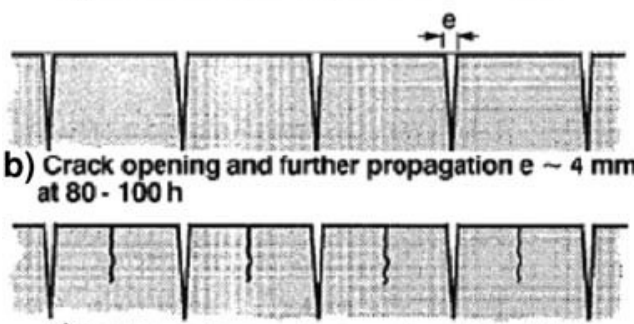

C) Initiation of secondary cracks at $80-100 \mathrm{~h}$

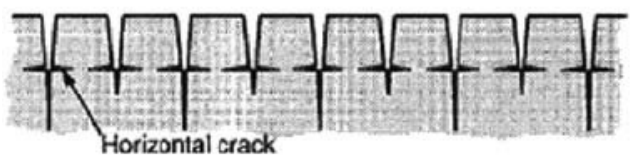

d) Differential shrinkage - Horizontal crack propagation in mode II (in plane shear) at $100-150 \mathrm{~h}$

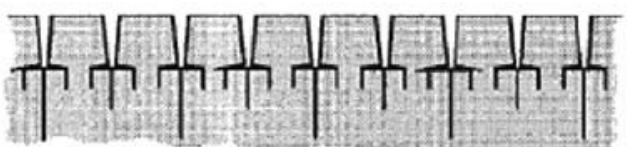

e) Initiation of cracks below shear plane at $150 \mathrm{~h}$

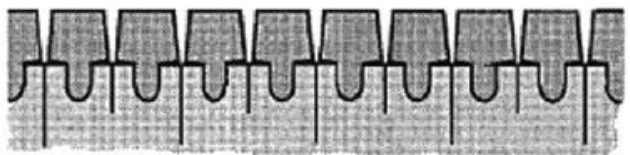

f) Formation of protuberance at $\mathrm{l}>192 \mathrm{~h}$

Fig. 6 Suggested mechanisms in play during the desiccation of the intact Saint-Alban clay (Konrad and Ayad 1997)

\section{Results and discussion}

Figure 6 shows the schematic description presented by Konrad and Ayad (1997) of the mechanisms leading to vertical cracks below shear plane and formation of protuberance. Within each polygon, vertical crack surfaces are initiated at a spacing of about one-third of the polygon width. Finally, continued evaporation and volume change leads to the formation of the observed protuberance in each polygon. The crack depth in the soil below the horizontal shear plane is thus reflected by the size of the protuberance and ranges between 4 and $6 \mathrm{~cm}$.

As it was mentioned before, the Wenner-Schlumberger method was used to measure the resistivity changes in the soil during the desiccation process.

The experiments were carried out in the same spirit as during a field survey on a real embankment as it is the final goal. The numbering and detection of vertical crack was the main target taking into account the limitation of the scanning method which was the electrodes geometry as the spacing kept between them was the same as the contact depth into the soil.

The first scan was taken after the clay compaction and before drying in order to confirm that the model was homogenous and was compacted to the expected state. The baseline map presented in Fig. 7, shows that the compaction was fully achieved and the measured resistivity was in the range of $20-30 \mathrm{ohm} \mathrm{m}$. This can be seen as a deep-blue color, which is the resistivity contour obtained after inversion of the experimental measurements.

a) Initial scan before drying
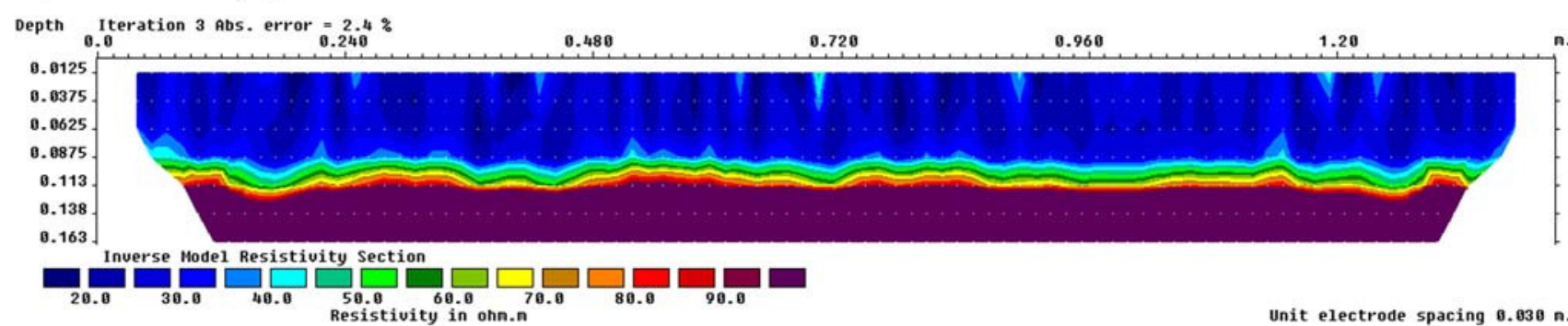

Unit electrode spacing $8.838 \mathrm{n}$.

b) After 4 hours of drying

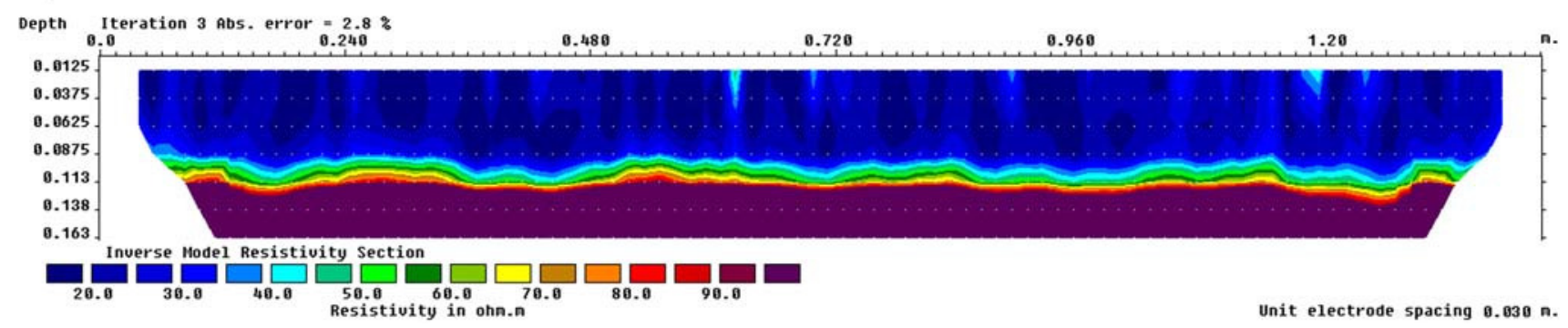

Fig. 7 Inverted resistivity baseline map using Res2DINV taken before drying process and after $4 \mathrm{~h}$ of heating 
C) After 20 hours of drying

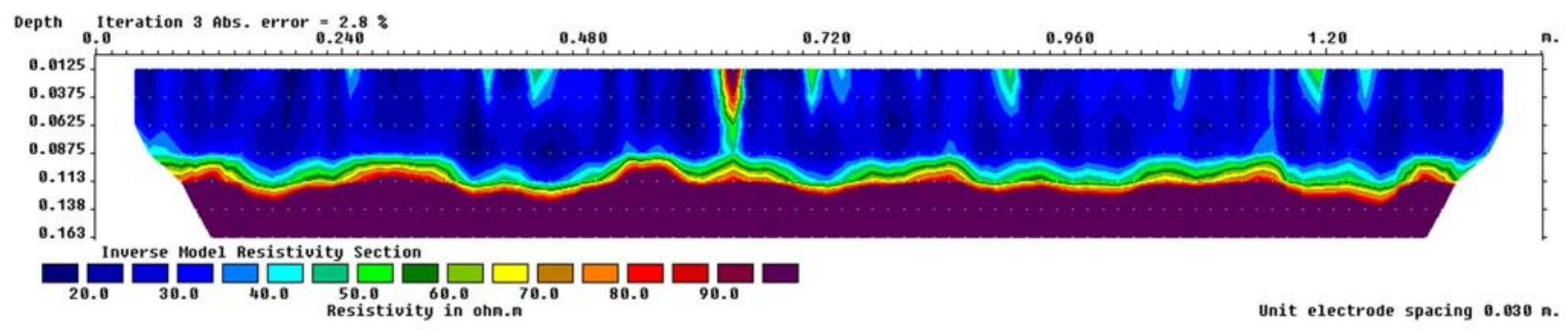

d) After 27 hours of drying

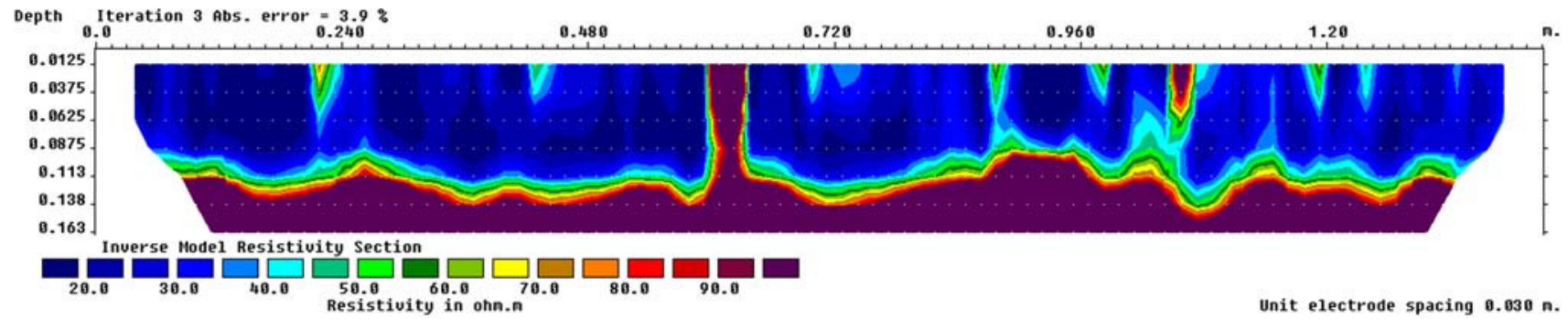

Fig. 8 Comparison between two measurements taken after 20 and $27 \mathrm{~h}$ of drying

e) After 43 hours of drying

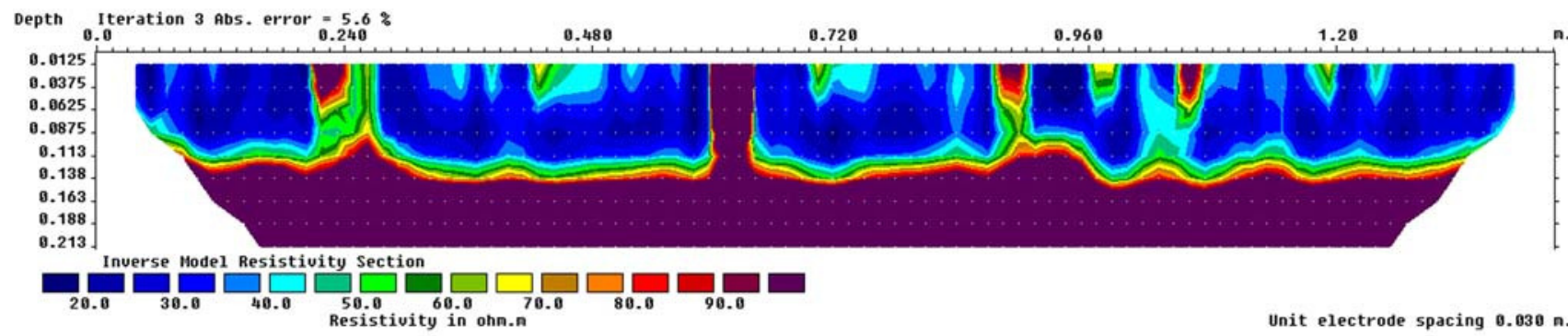

f) After 50 hours of drying

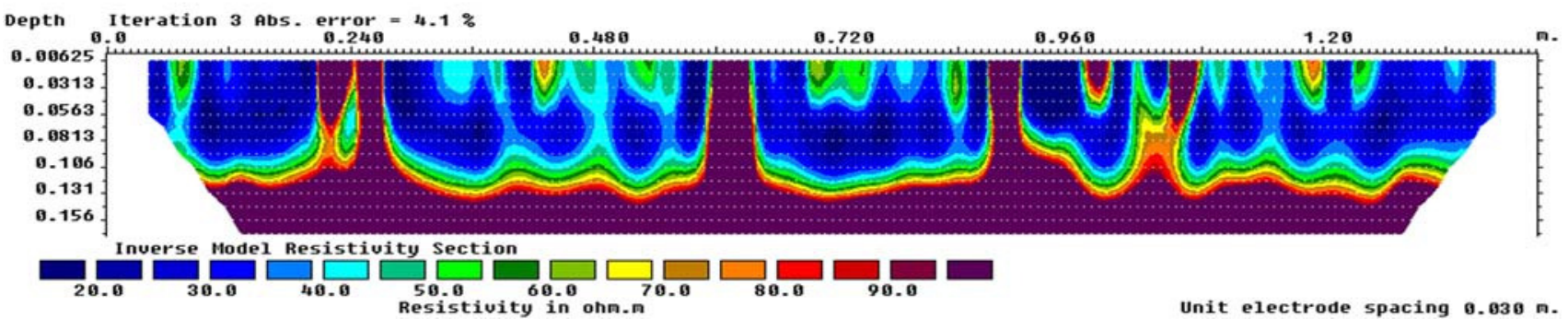

Fig. 9 Comparison between two measurements taken after 43 and $50 \mathrm{~h}$ of drying

A visual observation of the clay surface after the first $4 \mathrm{~h}$ is shown in Fig. 7. The temperature was increased from 15.0 up to $37.0^{\circ} \mathrm{C}$. The heat generated from the infra-red lamp, generated a temperature of $35.3^{\circ} \mathrm{C}$ above the model, similar to a very hot summer day.

The resistivity measurements were taken every morning, before the IR heater was switched on and every afternoon after the drying was finished. Due to the small changes in readings caused by evaporation process only morning's measurements were analyzed.

Figures 8 and 9 show how the resistivity changed with time and with cracks formation.

It can be clearly seen on the resistivity profile presented on Figs. 8 and 9 that several vertical openings occurred and 


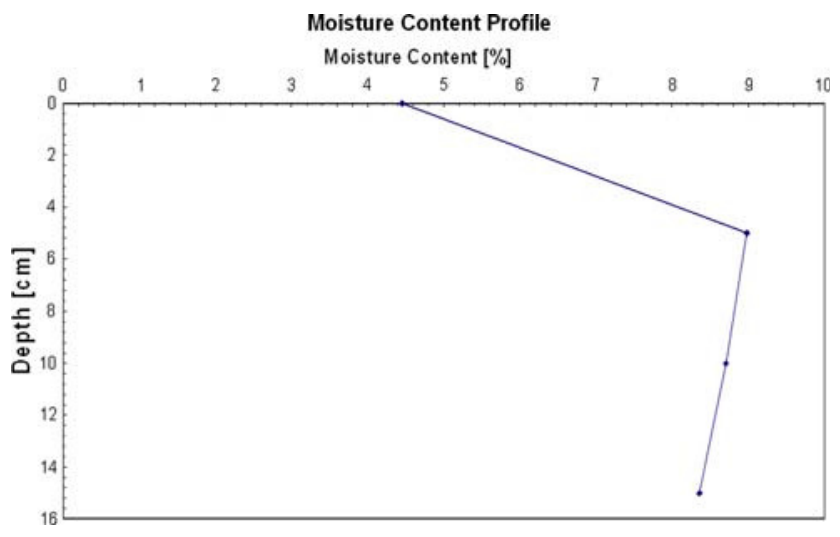

Fig. 10 Moisture content profile of the clay model used

became wider with time (vertical purple channels) corresponding visually to the same vertical cracks location on the laboratory clay sample.

However, it can be explained that during the fast heating process, the decrease in soil resistivity may be due to two phenomenon. The first can be described as micro swelling of the clay and closing up of the cracks caused by evaporating water represented by the disappearance of the light green vertical afternoon contours shown in Fig. 9. The second phenomenon could be due to the moisture content redistribution in the model due to evaporation and the water rising to the surface from deeper regions. A post mortem excavation of the model, and measurements of the moisture content at different depths, have shown a good agreement with the second assumption. The moisture content in the sample was between 8 and $9 \%$ below $5 \mathrm{~cm}$ depth and decreased sharply in the top $5 \mathrm{~cm}$ where the cracks developed. (Fig. 10)

The inversed resistivity map, shown of Fig. 11, has confirmed that the air space created inside the crack should give the high resistivity response in analyzed measurements shown as vertical purple (dark) wide channels. It has to be noted that the photographic picture of the cracks was made of 3 different pictures and was not at the same scale as the resistivity profile. The corresponding location of the cracks was given as an indication.

The resistivity results seem to reflect well the real desiccation cracking by comparing them with visual observations of the clay model.

One of the assumptions, which have been considered during this study, is that horizontal cracks can be observed as an horizontal discontinuity, between two layers and in this case should result as high resistivity measurements detected above an horizontal crack, as it is shown in Fig. 12. It is difficult to verify this assumption as the resistivity artifact due to the tank boundaries and shown before on Fig. 4 is also present.

This hypothesis can be explained as the shielding or air insulation for the current to pass through the horizontal discontinuity in the homogeneous soil which is the natural obstacle for the electrodes installed above it. The results are the creation of electrical anomalies as shown in Fig. 12. It is different for the electrodes positioned outside the range of the horizontal crack length, because current can go underneath it and the resistivity can be measured as before.

Use of viscous tracer for time detection improvement

The purpose of this experiment was to use a "slow" tracer, which can be injected into the cracks to improve the quality of the measurements, time related.

Because of the duration of the geo-electrical scans it was very important to find a tracer that could be viscous enough to let the time to the equipment to follow its seepage

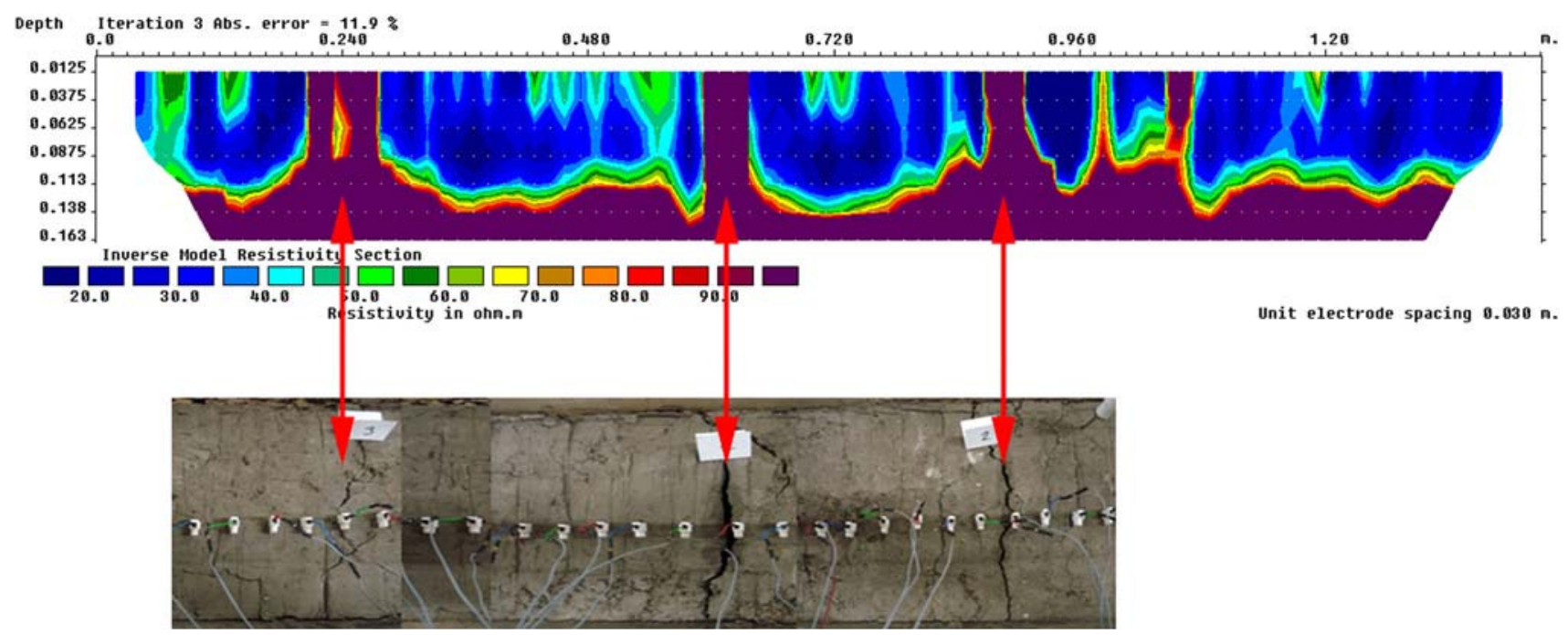

Fig. 11 Inverted resistivity map with corresponding picture of the cracked surface 
Fig. 12 Proposed interpretation for horizontal crack detection. Example of two points on picture (a) corresponding to the measurements shown in the cross section (b) and inverted to the resistivity contour model (c) a)
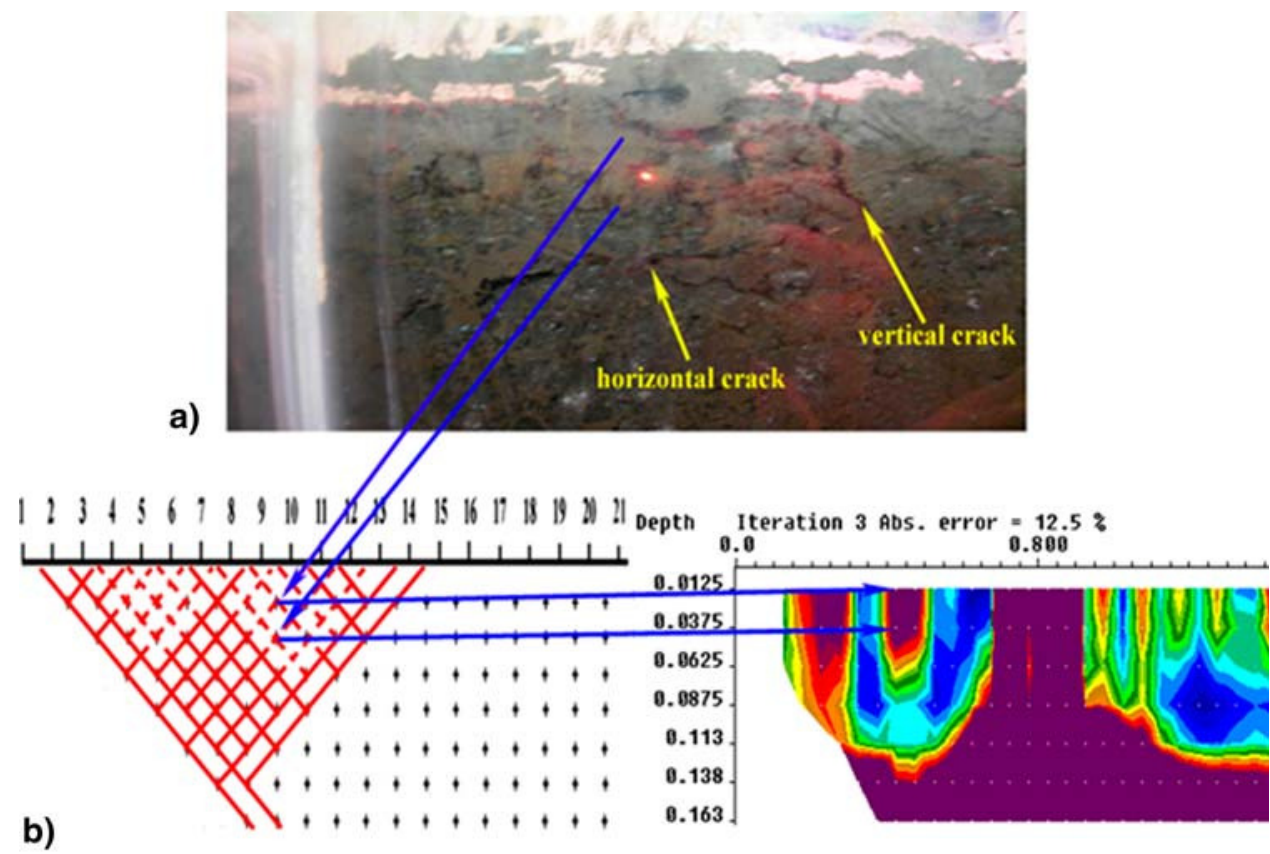

c)

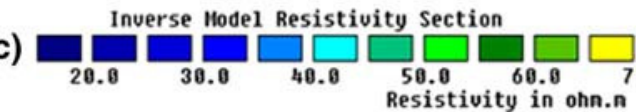

though the cracks. Another requirement for the tracer was to be able to penetrate the cracks remaining on the walls without any absorption by clay, which could significantly change the measurements.

It was decided to use the gelatinous substance, chiefly used as a solid substrate to contain culture medium for microbiological work. This substance called Plate Agar at a concentration of $8 \mathrm{~g} / \mathrm{l}$, was first dissolved in hot water and than $1 \%$ of $\mathrm{NaCl}$ was added to rise its electrical conductivity from 0.02 to $18.6 \mathrm{mS} / \mathrm{m}$.

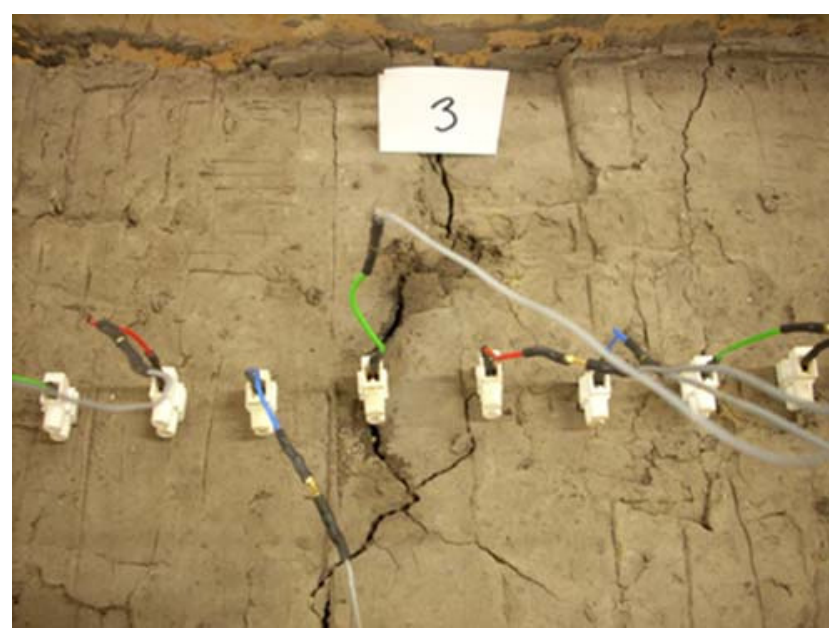

Fig. 13 Crack 3 where the Agar gel was injected alongside other cracks
Hence Agar mixed with $1 \%$ of $\mathrm{NaCl}$ was selected because the viscosity can be adjusted during the mixing. As Agar settles down at $46^{\circ} \mathrm{C}$ and then becomes a jelly substance too viscous to spread efficiently into the cracks it was very important to inject it into the crack in a state where it will flow freely, just after preparation.

This tracer was injected in a chosen crack in the experimental model (see Fig. 13).

The conductivity of the gel used was also measured before injection, to check the difference between higher resistive locations such as air pockets and cracks filled with the conductive substances.

As shown in Fig. 14, the scan taken after injection revealed the biggest changes in resistivity. The significant changes in the contour, from dark purple to deep blue as indicated by the circle on Fig. 14, showed that resistivity of the soil has dramatically decreased, and that the injected substance has mostly penetrated all surrounding area. It can be also seen that, in the place where horizontal crack was formed, the deep purple color started changing to a red line boundary indicated by arrows on Fig. 14, which could mean that air void was also filled with injected gel.

\section{Conclusions}

This experimental study has shown that a miniature geoelectrical method using resistivity arrays can be used as non-invasive method for the detection of desiccation cracks. 


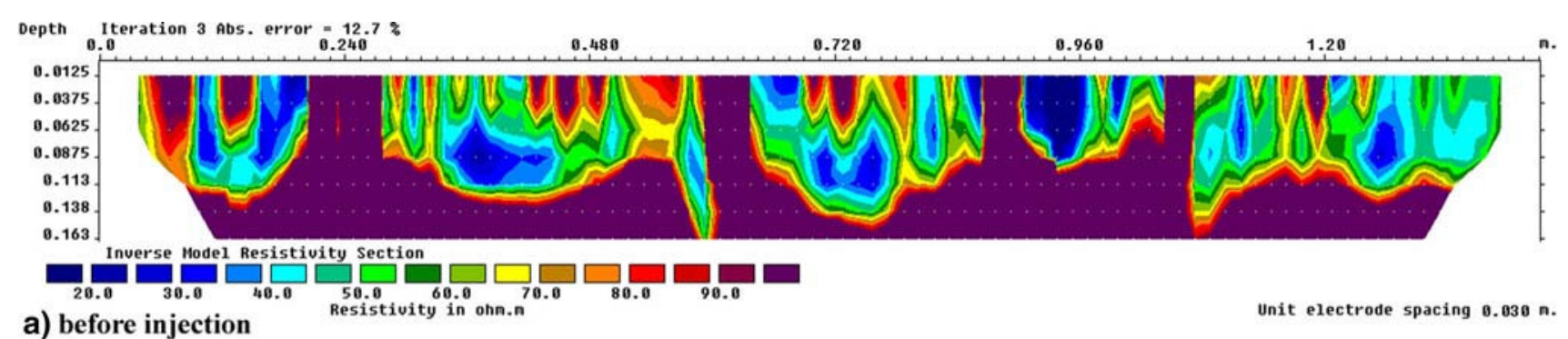

a) before injection

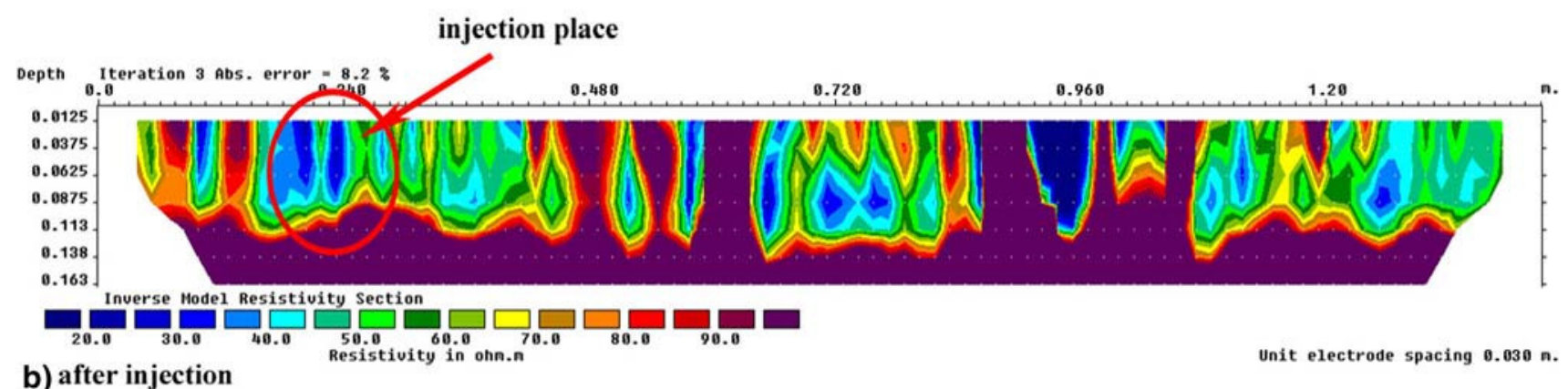

b) after injection

Fig. 14 Inverted resistivity scans for plate agar mixed with $1 \%$ of $\mathrm{NaCl}$ : a before injection, $\mathbf{b}$ after injection

All the major vertical cracks, which have been visually observed at the surface of the clay model, have been recorded by the resistivity equipment and displayed using the two-dimensional contour model software Res2Dinv. Despite the limitations of the method explained before, the vertical cracking network detected by the miniature resistivity arrays has been identified and validated by visual observations and post mortem examinations.

Assumptions were made about the detection of horizontal cracks that could be hampered by the insulating property of air related to crack continuity. The Agar gel results confirmed the boundaries of the cracks obtained from the previous results and delimited further the selected horizontal crack where the gel was injected. It is also very important to remember that electrical resistivity images are the outcome of data processing (i.e. they are based on apparent resistivity values) and for this reason they must not be interpreted as a direct representation of the field situation, but rather as a guide for qualitative estimation of the electrical resistivity distribution in the soil model. The limitation of the method was the geometry of the electrodes and boundary effects that will be necessary to investigate further to fully map the structure of the cracks also along the horizontal pattern in the future experiments on the scaled embankment built in our laboratory.

Acknowledgments Authors would acknowledge Francis McGillian, Antonio Montiaro, Ron Baron and Matthew Russell for their technical work and advice given to carry out this study.

\section{References}

Augier F et al (2002) On the risk of cracking in clay drying. Chem Eng J 86(1-2):133-138

Barker RD (1997) Electrical imaging and its application in engineering applications. Mod Phys Eng Geol 12:37-43

Chertkov VY, Ravina I (1998) Modelling the crack network of swelling clay soils. Soil Sci Soc Am J 62:1162-1171

Cooling LF, Marsland A (1954) Soil mechanics studies in the sea defense banks of Essex and Kent. In: Proceedings of the ICE conference on the north sea floods of 31 January/1 February 1953

Depountis N, Harris C, Davies MCR (1999) The application of miniaturised electrical imaging in scaled centrifuge modelling of pollution plume migration. In: Proceedings of 2 nd BGS international geoenvironmental engineering conference, London, pp 214-221

Griffiths DH, Barker RD (1993) Two-dimensional resitivity imaging and modelling in areas of complex geology. J Appl Geophys 29:211-226

Konrad JM, Ayad R (1997) Desiccation of a sensitive clay: field experimental observations. Can Geotech J 34:929-942

Lataste JF, Sirieix C, Breysse D, Frappa M (2003) Electrical resistivity measurement applied to cracking assessment on reinforced concrete structures in civil engineering. NDT \& E Int 36(6):383-394

Lecocq N, Vandewalle N (2003) Dynamics of crack opening in a onedimensional desiccation experiment. Phys A 321(3):431-441

Marsland A (1968) The Shrinkage and fissuring of clay in flood banks. Building research establishment, internal report No. 39/68

Moore PJ, Hor AYT (1984) Cracking behaviour of compacted clay. In: Proceedings of 4th Australia-New Zealand conference on geomechanics, Perth(2), pp 569-573

Nahlawi H, Kodikara J (2006) Laboratory experiments on desiccation cracking of thin soil layers. Geotech Geol Eng 24(6):1641-1664

Nguyen $F$ et al (2005) Image processing of 2D resistivity data for imaging faults. J Appl Geophys 57(4):260-277 
Rayhani MH, Yanful EK, Fakher A (2007) Desiccation-induced cracking and its effect on the hydraulic conductivity of clayey soils from Iran. Can Geotech J 44(3):276-283

Samouëlian A et al (2003) Electrical resistivity imaging for detecting soil cracking at the centimetric scale. Soil Sci Soc Am J 67(5):1319-1326
Tabbagh J, Samouëlian A, Cousin I (2007) Numerical modelling of direct current electrical resistivity for the characterisation of cracks in soils. J Appl Geophys 62(4):313-323

Towner GD et al (1987) The mechanics of cracking of drying clay. J Agri Eng Res 36(2):115-124 